\title{
Cellular apoptosis: an alternative mechanism of action of caspofungin in Candida glabrata
}

\author{
Maryam Moazeni ${ }^{1,2}{ }^{*}$,Parisa Aryamloo ${ }^{3}$, Hossein Asgarian-Omran ${ }^{4,5}$, Narges aslani ${ }^{6}$, \\ Hadi Hossein-Nataj ${ }^{4}$, Tahereh Shokohi ${ }^{1,2}$, Hamid Badali ${ }^{1,2}$, Mojtaba Nabili ${ }^{7}$
}

${ }^{1}$ Invasive Fungi Research Centre, Mazandaran University of Medical Sciences, Sari, Iran; ${ }^{2}$ Department of Medical Mycology, School of Medicine, Mazandaran University of Medical Sciences, Sari, Iran; ${ }^{3}$ Student Research Committee, Mazandaran University of Medical Sciences, Sari, Iran; ${ }^{4}$ Department of Immunology, School of Medicine, Mazandaran University of Medical Sciences, Sari, Iran; 5 Immunogenetics Research Centre, School of Medicine, Mazandaran University of Medical Sciences, Sari, Iran; ${ }^{6}$ Cellular and Molecular Research Center, Urmia University of Medical Sciences, Urmia, Iran; ${ }^{7}$ Department of medical laboratory sciences, Sari Branch, Islamic Azad University, Sari, Iran

Introduction: Although the mechanism of activity for echinocandins is known, the physiological mechanisms by which these antifungal agents cause cell death via the classical apoptotic pathways are not well-defined yet. Regarding this, the present study aimed to evaluate the mechanisms of caspofungin-induced Candida glabrata cell death.

Material and methods: For the purpose of the study, the minimum inhibitory concentration (MIC) of caspofungin against C. glabrata (ATCC 90030) was determined using the broth microdilution reference method (CLSI M27-A2 and M27-S4). The annexin $\mathrm{V}$ and propidium iodide staining was performed to determine the way through which caspofungin exerts activity against $C$. glabrata (i.e., through the induction of apoptosis and/or necrosis). Additionally, the possible effect of caspofungin on inducing the expression of two apoptotic genes, namely MCA1 and NUC, was studied using the real-time polymerase chain reaction assay.

Results: According to the obtained MIC (0.5 $\mu \mathrm{g} / \mathrm{mL}), C$. glabrata, exposed to $0.25,0.5$, and $1 \mu \mathrm{g} / \mathrm{mL}$ of caspofungin, exhibited the features of late apoptosis/necrosis after $18 \mathrm{~h}$ of incubation. Furthermore, the use of $0.25,0.5$, and $1 \mu \mathrm{g} / \mathrm{ml}$ caspofungin induced apoptosis (early/late) in 14.67\%, 17.04\%, and $15.89 \%$ of the cells, respectively. There was a significant difference between the percentages of early-apoptotic cells at the three concentrations $(P<0.05)$. The rate of necrosis was significantly greater in response to caspofungin. Accordingly, necrosis occurred in $71.26 \%, 71.26 \%$, and $61.26 \%$ of the cells at
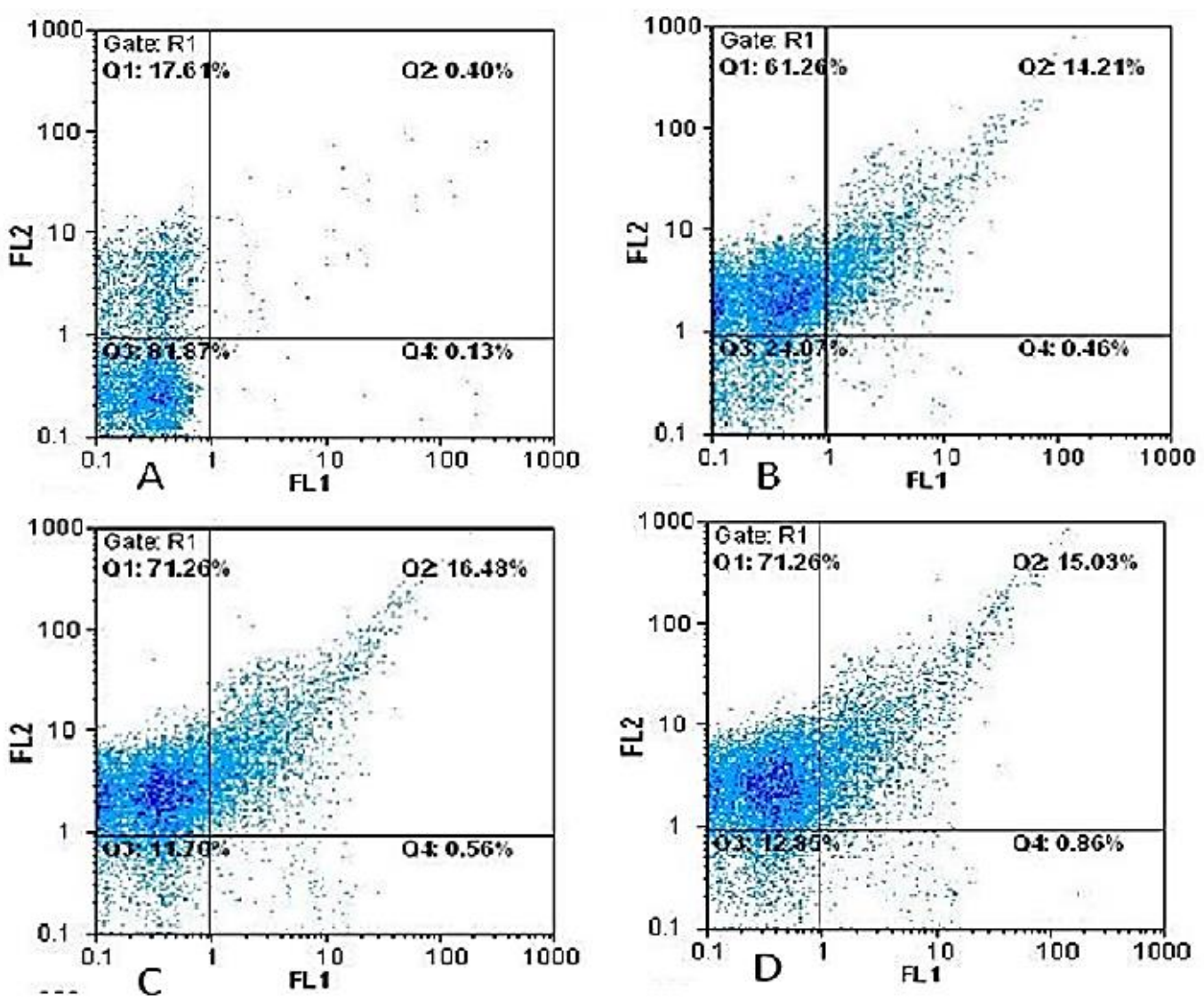

the caspofungin concentrations of $0.25,0.5$, and $1 \mu \mathrm{g} / \mathrm{mL}$, respectively $(\mathrm{P}<0.05)$. The analysis of the data in REST software demonstrated that the expression of MCA1 and NUC1 genes had a significant increase $(P<0.05)$.

Conclusion: As the findings of the present study demonstrated, caspofungin promoted both necrosis and apoptosis of $C$. glabrata cells at concentrations higher or equal to the MIC.

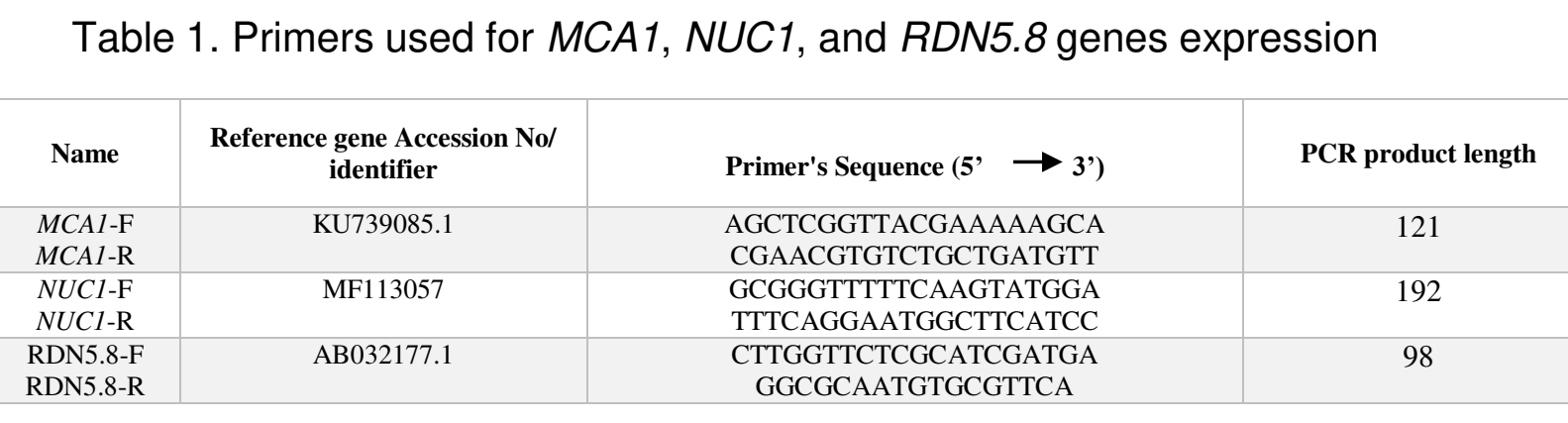

Table 2. Effects of caspofungin on early apoptosis, late apoptosis, and necrosis, as determined by annexin $\mathrm{V}$ and propidium iodide staining

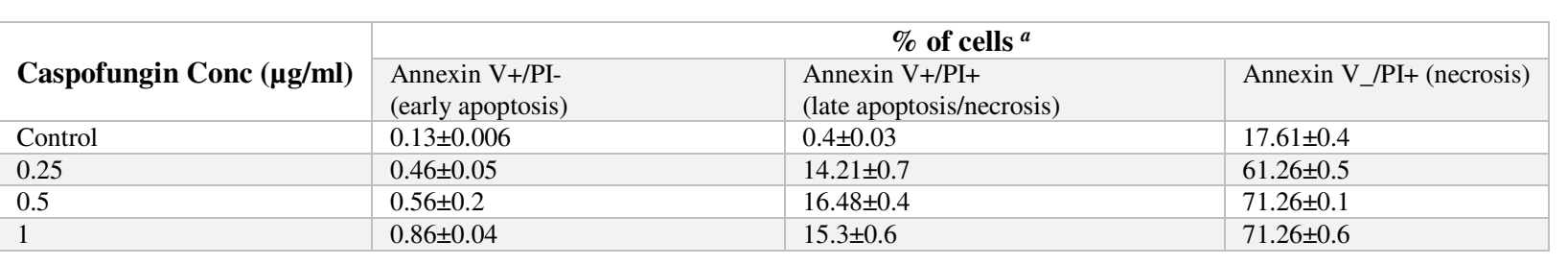

Table 3. Expression pattern of apoptosis genes in standard isolate of $C$. glabrata

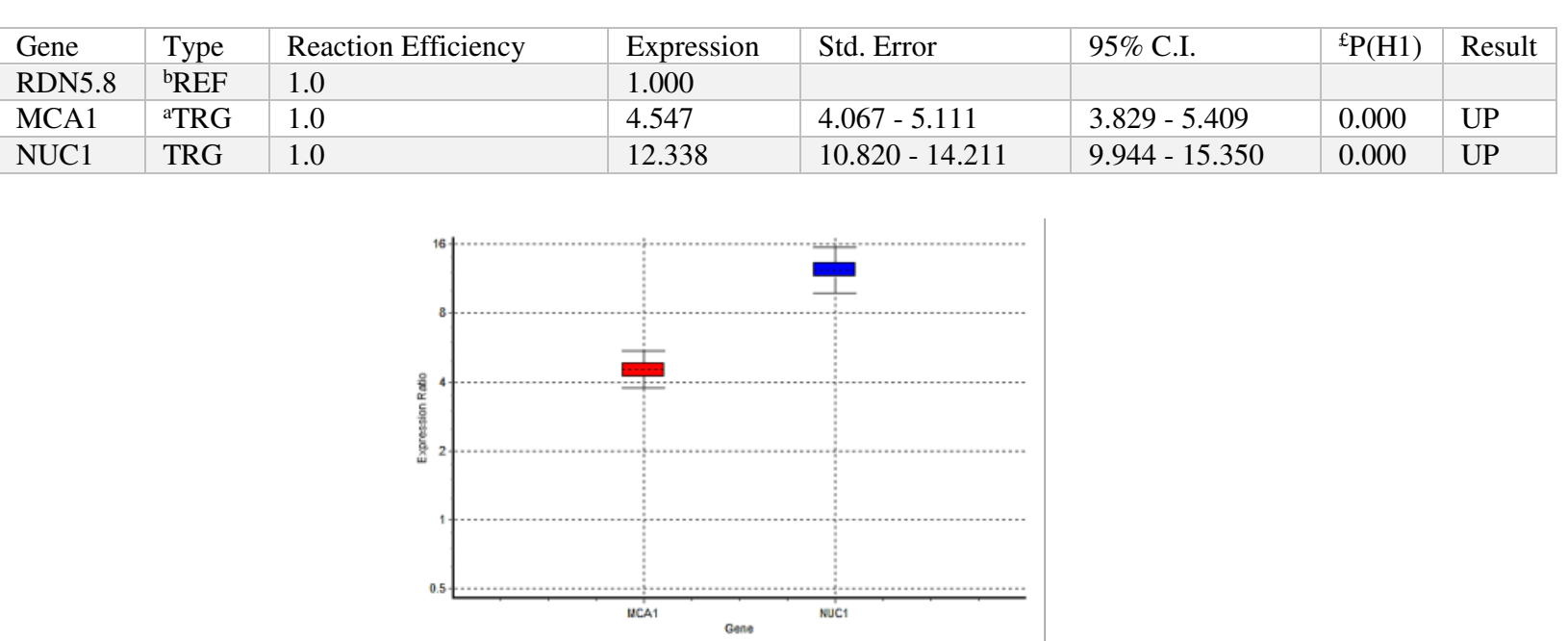

Ratio of the expression of two apoptotic major genes. MCA1 and NUC1 are representative for caspase-dependent and caspase-independent apoptosis induction in yeast, respectively.

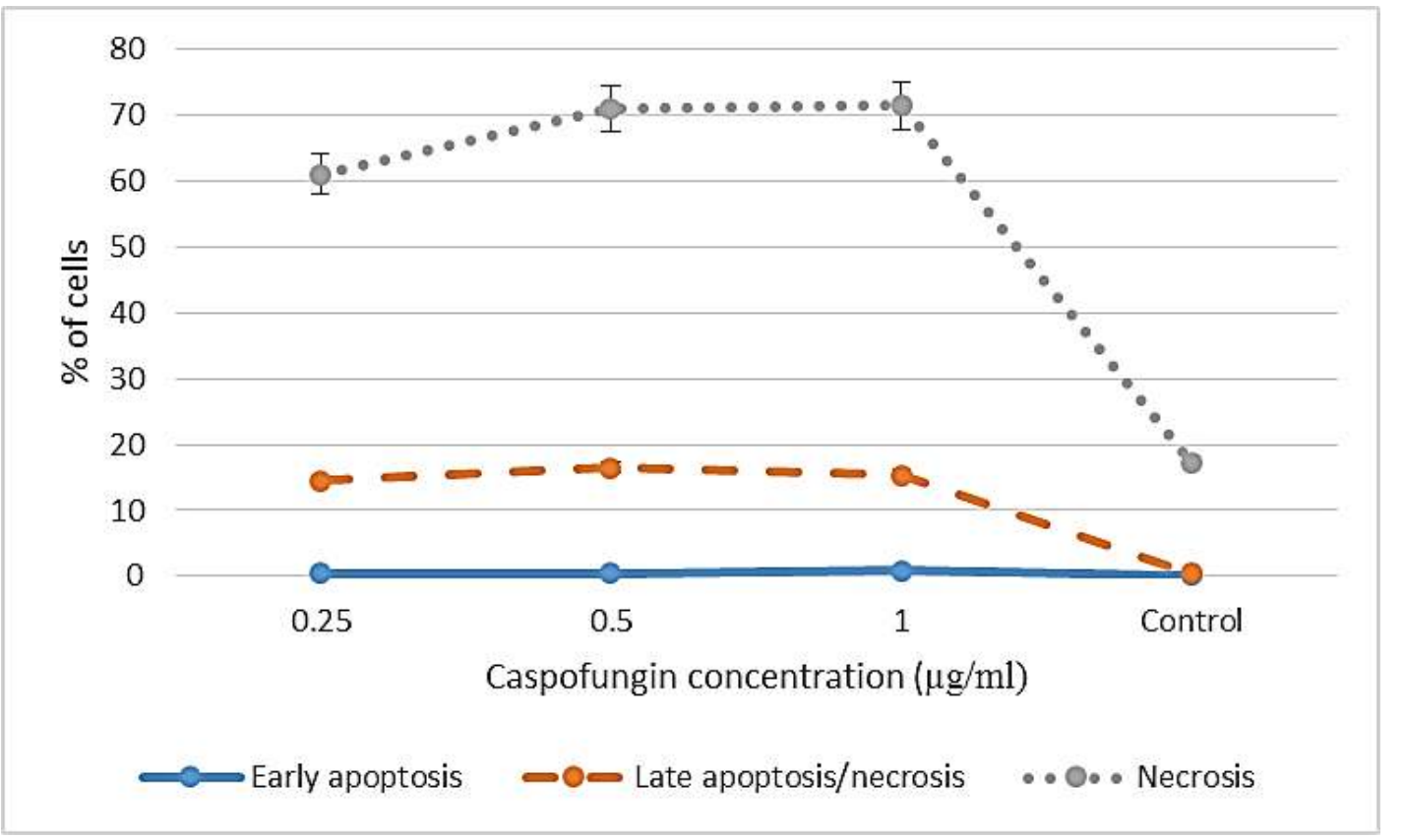

Chart 1. Effects of different concentration of caspofungin on early apoptosis, late apoptosis, and necrosis, as determined by annexin $\mathrm{V}$ and propidium iodide staining after $18 \mathrm{hr}$ of incubation 